\title{
Recent results within Lipatov's high energy effective action
}

\section{Grigorios Chachamis}

Instituto de Física Corpuscular UVEG/CSIC, E-46980 Paterna (Valencia), Spain

E-mail: Grigorios.Chachamiseific.uv.es

Martin Hentschinski*

Physics Department, Brookhaven National Laboratory, Upton, New York 11973, USA

E-mail: hentsch@bnl.gov

\section{Jose Daniel Madrigal Martínez}

Instituto de Fśica Teórica UAM/CSIC, C.U. Cantoblanco, E-28049 Madrid, Spain.

E-mail: josedaniel.madrigal@uam.es

\section{Agustín Sabio Vera}

Instituto de Fśica Teórica UAM/CSIC, C.U. Cantoblanco, E-28049 Madrid, Spain.

E-mail: sabiodcern.ch

We review Lipatov's high energy effective action and show that it is a useful computational tool to calculate QCD scattering amplitudes in the high energy limit. We explain in some detail our recent work where a novel regularization and subtraction procedure has been proposed that allows to extend the use of this effective action beyond tree level. As explicit results we discuss the derivation of forward jet vertices, for jet events with and without rapidity gaps.

XXI International Workshop on Deep-Inelastic Scattering and Related Subject-DIS2013, 22-26 April 2013

Marseilles, France

\footnotetext{
* Speaker.
} 


\section{Introduction}

Due to its large center of mass energy the LHC provides an ideal opportunity to test BFKLdriven observables [1,2]. Among them both central production processes, such as heavy quark production [3], forward production of high $p_{T}$ jets [4] and Drell-Yan pairs [5, 6, 7, 8], and processes with hard events in both forward and backward direction, i.e. forward-backward ('MuellerNavelet') jets [9, 10] and forward $Z$ boson production combined with a backward jet [11].

An observable of particular interest is given by forward/backward jets with a rapidity gap ('Mueller-Tang' jets). This process is special since it allows to probe the non-forward BFKL kernel, unlike the previously mentioned processes restricted to the forward case. From a phenomenological point of view the description in terms of the non-forward BFKL Green's function is of relevance, since the latter describes a color singlet $t$-channel exchange. Unlike configurations which merely suppress emissions above a certain veto scale, the non-forward BFKL Green's function describes a $t$-channel exchange with emissions into the gap region intrinsically absent.

While the non-forward BFKL kernel is currently available at next-to-leading order (NLO) [12], only the virtual NLO corrections to the impact factors [15] are currently known; phenomenological studies, see e.g. $\quad[13,14]$, are therefore limited to leading order (LO) impact factors. As NLO corrections to BFKL observables are often found to be size-able, this limitation to LO impact factors is currently one of the main drawbacks of BFKL phenomenology. A powerful tool to overcome this limitation is given by Lipatov's effective action [16]. It is given in terms of the conventional QCD action to which a new induced term is added. The latter contains a new effective degree of freedom, the reggeized gluon, which has been introduced in order to achieve a gauge invariant factorization of QCD amplitudes in the high energy limit. The determination of higher order corrections within this effective action is at first plagued by both technical and conceptual difficulties. Loop corrections show a new type of divergence, which is not present in usual QCD Feynman diagrams. Supplementing the QCD action with the additional induced term leads to an apparent over-counting problem. These problems have been addressed and resolved recently, first in the context of LO transition kernels [17, 18, 18, 20, 19] and later on in the calculation of NLO corrections to the forward quark and gluon -initiated jet vertex [21, 22] and the quark and gluon contribution to the two-loop gluon trajectory [23], for a recent review see [24].

In this contribution we present some details of the determination of the missing real NLO correction to the quark-initiated Mueller-Tang jet impact factors. In particular we address the implementation of an alternative implementation of an rapidity regulator, needed in the evaluation of real corrections. While Sec. 2 contains some general details on the effective action, Sec. 3 discusses different realizations of regulators in the case of Mueller-Navelet jets. For details we refer to [25].

\section{The high energy effective action}

The effective action adds to the QCD action an induced term, $S_{\mathrm{eff}}=S_{\mathrm{QCD}}+S_{\text {ind. }}$, which describes the coupling of the reggeized gluon field $A_{ \pm}(x)=-i t^{a} A_{ \pm}^{a}(x)$ to the usual gluonic field 
$v_{\mu}(x)=-i t^{a} v_{\mu}^{a}(x)$. This induced term reads

$$
\begin{aligned}
S_{\text {ind. }}\left[v_{\mu}, A_{ \pm}\right]=\int \mathrm{d}^{4} x \operatorname{tr}\left[\left(W_{+}[v(x)]-A_{+}(x)\right) \partial_{\perp}^{2} A_{-}(x)\right] \\
+\int \mathrm{d}^{4} x \operatorname{tr}\left[\left(W_{-}[v(x)]-A_{-}(x)\right) \partial_{\perp}^{2} A_{+}(x)\right] .
\end{aligned}
$$

The infinite number of couplings of the gluon field to the reggeized gluon field are encoded in two functionals $W_{ \pm}[v]=v_{ \pm} \frac{1}{D_{ \pm}} \partial_{ \pm}$where $D_{ \pm}=\partial_{ \pm}+g v_{ \pm}$. Reggeized gluon fields are invariant under local $\mathrm{SU}\left(N_{c}\right)$ gauge transformations. Strong ordering of longitudinal momenta in high energy factorized amplitudes provides the following kinematic constraint,

$$
\partial_{+} A_{-}(x)=\partial_{-} A_{+}(x)=0
$$

which is always implied. Quantization of the gluonic field requires to add gauge fixing and ghost terms, which we have included in the QCD action. Feynman rules are given in Fig. 1. Curly lines

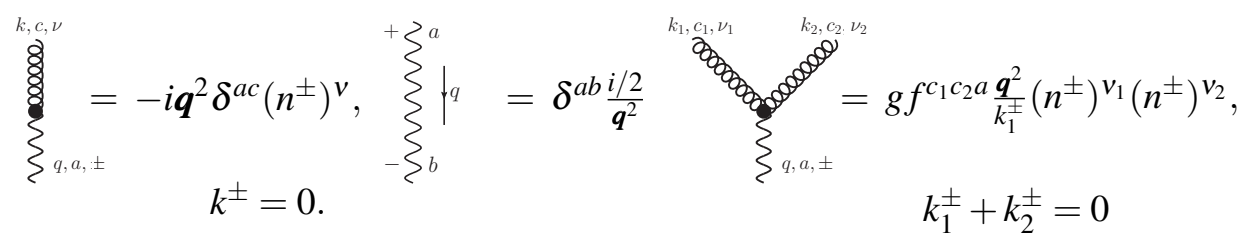

Figure 1: From left to the right: the direct transition vertex, the reggeized gluon propagator and the order $g$ induced vertex.

describe the conventional QCD gluon field and wavy lines the reggeized gluon field. There exist an infinite number of higher order induced vertices. For the present analysis only the order $g$ induced vertex in Fig. 1 is needed. Loop corrections furthermore require a regularization of the light-cone singularity $1 / k_{1}^{ \pm}$. As discussed in [26] this pole should be treated as a Cauchy principal value.

\section{Rapidity regulator and the Mueller-Navelet jet}

The real corrections to the quark induced Mueller-Navelet jet have been derived from the effective action in [21]. They can be organized into three contributions to the five-point amplitude $q\left(p_{a}\right)+q\left(p_{b}\right) \rightarrow q\left(p_{1}\right)+g(q)+q\left(p_{2}\right)$, i.e. central and quasi-elastic gluon production process, see Fig. 2. The central production amplitude, obtained from the sum of the following three effective
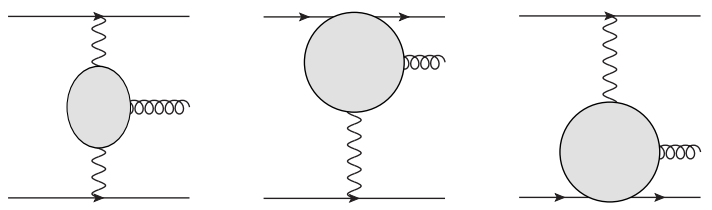

Figure 2: Gluon production at central rapidities and in the fragmentation region of the initial quarks 
diagrams, yields the unintegrated real part of the forward LO BFKL kernel:

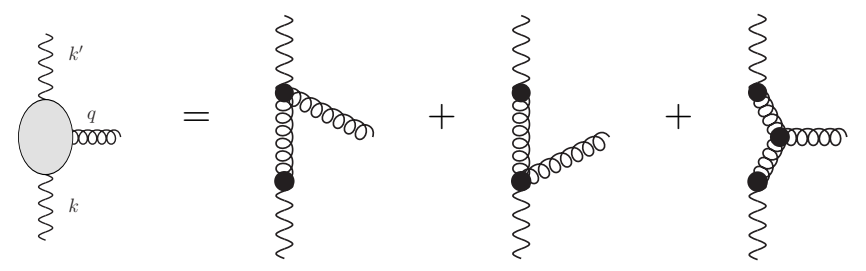

It leads to the following production vertex

$$
V\left(\boldsymbol{q} ; \boldsymbol{k}, \boldsymbol{k}^{\prime}\right)=\frac{N_{c}^{2}-1}{8(2 \pi)^{3+2 \varepsilon} \boldsymbol{k}^{2} \boldsymbol{k}^{\prime 2}} \overline{|\mathscr{M}|^{2}}{ }_{r^{*} r^{*} \rightarrow g}=\frac{\alpha_{s} N_{c}}{\mu^{2 \varepsilon} \pi^{2+\varepsilon} \boldsymbol{q}^{2}} .
$$

The exclusive differential cross section for central production then reads

$$
d \hat{\sigma}_{a b}^{(c)}=h_{a}^{(0)}\left(\boldsymbol{k}^{\prime}\right) h_{b}^{(0)}(\boldsymbol{k}) V\left(\boldsymbol{q} ; \boldsymbol{k}, \boldsymbol{k}^{\prime}\right) d^{2+2 \varepsilon} \boldsymbol{k}^{\prime} d^{2+2 \varepsilon} \boldsymbol{k} d \eta
$$

For the quasi-elastic contribution $q\left(p_{a}\right) r^{*}(k) \rightarrow g(q) q(p)$ we evaluate at first the sum of the effective diagrams

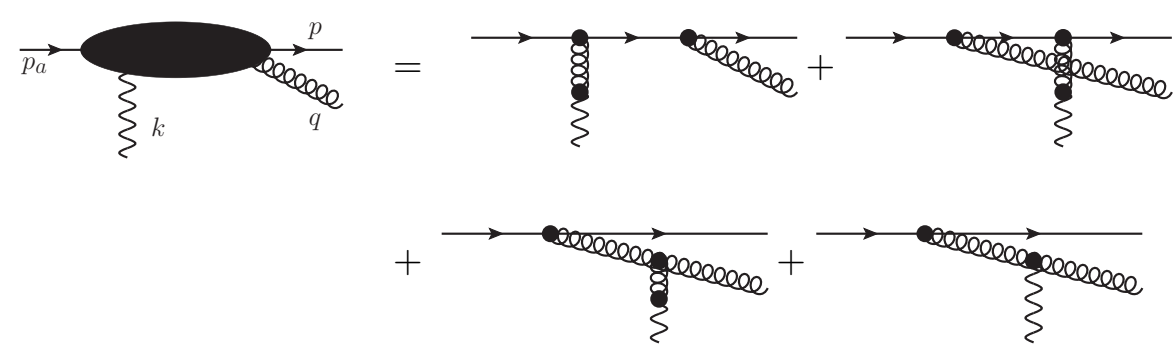

The final result exactly agrees with the equivalent one in [27]:

$$
\begin{aligned}
h^{(1)}\left(z ; \boldsymbol{k}^{2}, \boldsymbol{k}^{\prime 2}, \boldsymbol{q}^{2}\right) & =h^{(0)}\left(\boldsymbol{k}^{2}\right) \cdot \mathscr{F}_{q q g}(z, \boldsymbol{q}, \boldsymbol{k}), \\
\mathscr{F}_{q q g}(z, \boldsymbol{q}, \boldsymbol{k}) & =\frac{\alpha_{s}}{2 \pi} \frac{\mathscr{P}_{g q}(z, \boldsymbol{\varepsilon})}{\pi_{\varepsilon}} \frac{\left[C_{F} z^{2} \boldsymbol{k}^{\prime 2}+N_{c}(1-z) \boldsymbol{\Delta} \cdot \boldsymbol{q}\right]}{\boldsymbol{q}^{2} \boldsymbol{\Delta}^{2}},
\end{aligned}
$$

where $\mathscr{P}_{g q}(z, \varepsilon)=\frac{1+(1-z)^{2}+\varepsilon z^{2}}{z}$ is, up to a factor $C_{F}$, the real part of the $q \rightarrow g$ splitting function and $\boldsymbol{k}^{\prime}=\boldsymbol{q}-\boldsymbol{k}$. Note that in the limit $z \rightarrow 0$, which corresponds for fixed gluon transverse momentum $\boldsymbol{q}^{2}$ to a large difference in rapidity of final state quark and gluon, the above expression turns into the central production vertex, multiplied with the leading order impact factor

$$
\lim _{z \rightarrow 0} h^{(1)}\left(z ; \boldsymbol{k}^{2}, \boldsymbol{k}^{\prime 2}, \boldsymbol{q}^{2}\right)=h^{(0)}\left(\boldsymbol{k}^{2}\right) V\left(\boldsymbol{q} ; \boldsymbol{k}, \boldsymbol{k}^{\prime}\right)
$$

Adding quasi-elastic and central production cross-section leads therefore to the expected overcounting. For production processes at tree-level, there are two immediate solutions. A physical intuitive solution slices the longitudinal phase space and associates a fixed range of rapidity to gluon production in the quasi-elastic (where the rapidity separation of gluon and one final state 
quark is small) and central region (with significant rapidity separation from both quarks). As an alternative to such an treatment, which proves highly useful in the evaluations of virtual corrections, see [21, 23, 24], one subtracts Eq. (3.4) from the quasi-elastic correction, schematically

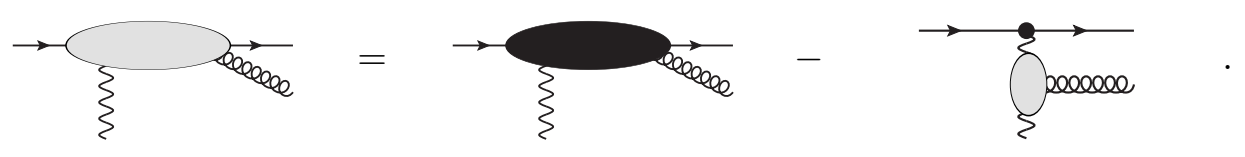

If integrated over rapidity, both central and quasi-elastic production vertex lead to a divergent integral. In $[21,22]$ this has been treated through introducing cut-offs on the gluon rapidity $\eta$, with $\eta_{a}>\eta>\eta_{b}$ for the central production vertex Eq. (3.2) and $\eta>v_{b}\left(\eta<v_{a}\right)$ for the quasi-elastic corrections in the fragmentation regions of the quarks with with momenta $p_{a}$ and $p_{b}$ respectively. Subtracting central from quasi-elastic corrections, the dependence on the regulators cancels for the sum of the three diagrams Fig. 2, if evaluated in the limit $\eta_{a, b} \rightarrow \pm \infty$.

In the case of Mueller-Tang jets on the other hand, where gluon emission is restricted to the fragmentation region of the initial quarks, not all cut-offs can be evaluated in the limit $\eta_{a, b} \rightarrow \infty$ which requires a careful fine-tuning of the cut-offs to avoid conflicts with collinear factorization. In such a case it is preferable to perform the subtraction Eq. (3.5) without any cut-off, leading to the entire elimination of the high energy divergence in the quasi-elastic correction. For MuellerNavelet jets, such as treatment results into the replacement

$$
\mathscr{F}_{q q g}(z, \boldsymbol{q}, \boldsymbol{k}) \rightarrow \mathscr{F}_{q q g}(z, \boldsymbol{q}, \boldsymbol{k})-\frac{\alpha_{s} N_{c}}{2 \pi \pi_{\varepsilon}} \frac{2}{z \boldsymbol{q}^{2}},
$$

in Eq. (3.3). The rapidity integral over the central production vertex is then on the other hand evaluated with the upper and lower bounds provided by the phase space in the fragmentation region of the two quarks. Both methods are entirely equivalent as long as one integrates over the entire phase space, the second method provides however a technical advantage if one desires to impose cuts on the phase space of the gluon, as it is the case for Mueller-Tang jets.

For further details, including the calculation of the NLO corrections to the Mueller-Tang impact factor at NLO we refer to the paper in preparation [25], some details are already contained in $[29,30]$.

\section{Acknowledgments}

We thank J. Bartels, V. Fadin and L. Lipatov for their constant support with useful discussions. We acknowledge support by the Research Executive Agency (REA) of the European Union under the Grant Agreement number PITN-GA-2010-264564 (LHCPhenoNet). G.C. acknowledges support from Marie Curie actions (PIEF-GA-2011-298582). M.H. acknowledges support from the U.S. Department of Energy under contract number DE-AC02-98CH10886 and a BNL "Laboratory Directed Research and Development" grant (LDRD 12-034).

\section{References}

[1] V. S. Fadin, E. A. Kuraev and L. N. Lipatov, Phys. Lett. B 60 (1975) 50. 
[2] I. I. Balitsky and L. N. Lipatov, Sov. J. Nucl. Phys. 28 (1978) 822 [Yad. Fiz. 28 (1978) 1597].

[3] G. Chachamis, M. Hentschinski, A. Sabio Vera and C. Salas, arXiv:0911.2662 [hep-ph].

[4] M. Deak, F. Hautmann, H. Jung and K. Kutak, Eur. Phys. J. C 72 (2012) 1982 [arXiv:1112.6354 [hep-ph]].

[5] F. Hautmann, M. Hentschinski and H. Jung, Nucl. Phys. B 865 (2012) 54 [arXiv:1205.1759 [hep-ph]].

[6] F. Hautmann, M. Hentschinski and H. Jung, arXiv:1209.6305 [hep-ph],

[7] F. Hautmann, M. Hentschinski and H. Jung, arXiv:1207.6420 [hep-ph],

[8] F. Hautmann, M. Hentschinski and H. Jung, arXiv:1205.6358 [hep-ph].

[9] B. Ducloue, L. Szymanowski and S. Wallon, JHEP 1305, 096 (2013) [arXiv:1302.7012 [hep-ph]].

[10] F. Caporale, B. Murdaca, A. Sabio Vera and C. Salas, arXiv:1305.4620 [hep-ph].

[11] M. Hentschinski, C. Salas, "Forward Drell-Yan and backward jet as a test of BFKL evolution", Proceedings of the XXth International Workshop on Deep-Inelastic Scattering and Related Subjects (DIS 2012), arXiv:1301.1227 [hep-ph].

[12] V. S. Fadin and R. Fiore, Phys. Rev. D 72 (2005) 014018 [hep-ph/0502045].

[13] O. Kepka, C. Marquet and C. Royon, Phys. Rev. D 83 (2011) 034036 [arXiv:1012.3849 [hep-ph]].

[14] R. Enberg, G. Ingelman and L. Motyka, Phys. Lett. B 524 (2002) 273 [hep-ph/0111090].

[15] V. S. Fadin, R. Fiore, M. I. Kotsky and A. Papa, Phys. Rev. D 61 (2000) 094006 [hep-ph/9908265].

[16] L. N. Lipatov, Nucl. Phys. B 452 (1995) 369 [hep-ph/9502308].

[17] M. Hentschinski, Nucl. Phys. Proc. Suppl. 198 (2010) 108 [arXiv:0910.2981 [hep-ph]].

[18] M. Hentschinski, arXiv:0908.2576 [hep-ph].

[19] M. Hentschinski, J. Bartels and L. N. Lipatov, arXiv:0809.4146 [hep-ph].

[20] M. Hentschinski, Acta Phys. Polon. B 39 (2008) 2567 [arXiv:0808.3082 [hep-ph]].

[21] M. Hentschinski and A. S. Vera, Phys. Rev. D 85 (2012) 056006 [arXiv:1110.6741 [hep-ph]].

[22] G. Chachamis, M. Hentschinski, J. D. Madrigal and A. S. Vera, arXiv:1212.4992 [hep-ph].

[23] G. Chachamis, M. Hentschinski, J. D. Madrigal Martinez and A. Sabio Vera, Nucl. Phys. B 861 (2012) 133 [arXiv:1202.0649 [hep-ph]], and article in preparation.

[24] G. Chachamis, M. Hentschinski, J. D. Madrigal Martinez and A. Sabio Vera, arXiv:1211.2050 [hep-ph].

[25] M. Hentschinski, J. D. Madrigal Martinez, B. Murdaca, C. Salas and A. Sabio Vera, in preparation.

[26] M. Hentschinski, Nucl. Phys. B 859 (2012) 129 [arXiv:1112.4509 [hep-ph]].

[27] M. Ciafaloni, Phys. Lett. B 429, 363 (1998) [hep-ph/9801322].

[28] A. H. Mueller and W. -K. Tang, Phys. Lett. B 284 (1992) 123.

[29] M. Hentschinski, B. Murdaca and A. S. Vera, arXiv:1206.1622 [hep-ph].

[30] M. Hentschinski and B. Murdaca, AIP Conf. Proc. 1523, 268 (2012). 